\title{
A Development of Practical Cost Control System for Small and Medium-sized Contractors
}

\author{
Vacharapoom Benjaoran, Anucha Kaewsikhoa, and Wisitsak Tabyang, Member, IACSIT
}

\begin{abstract}
A cost control of a construction project is a crucial management task which is a key to success of the business. It requires a number of up-to-date input data consistently throughout the construction phase. Small and medium sized contractors (SMCs) with limited personnel and investments normally fail to monitor and control their project costs. Sources of actual cost data are often scattered in various billing documents. The timeliness and the accuracy of these input data directly affect the effectiveness of the control task. The collection and preparation of the actual cost data become critical drawbacks. The Barcode-based Cost Control System (BCCS) is developed on the spreadsheet software for SMCs. BCCS compares the actual costs to-date against the budgets which are arranged from the bills of quantity and the common cost account structures. Results from the system development and verification with construction project cases find that BCCS can help reduce time-effort and input-errors compared to the manual method. It is an easy-to-use but efficient tool for SMCs to control their project costs.
\end{abstract}

Index Terms-Barcode, construction project control, cost control, small and medium-sized contractors.

\section{INTRODUCTION}

Controlling is one of the most important tasks of construction project management, not less than planning. Unless a project is continuously and carefully controlled throughout the project duration, the project may not be successful as planned. Planning is done based on data from estimation, prediction and planners own judgments whereas controlling is done based on actual data from the construction site. Hence, controlling requires consistent efforts and a plenty of actual data collected. It is carried out under the time-pressure to synchronize with the current progress on site.

A project cost control is an attempt to restrict the actual costs within the budgeted costs. Variances between the actual and budget costs must be quantified on each cost center. A proper corrective action will be carried out when any cost overrun is found [2]. Project costs are all expenditures that occur on construction site including both direct and indirect costs. The level of the control depends on the breakdown structure of project costs into the number of individual cost centers. A cost center is the finest unit of control. Any cost center must be consistently controlled. The actual cost of each cost center is cumulated over time. A cost overrun that is lately discovered may not be able to correct.

Particularly, a cost control consists of two major processes

Manuscript received May 7, revised June 1, 2012.

Authors are with School of Civil Engineering, Suranaree University of Technology, Nakhonratchasima, Thailand (e-mail: vacharapoom@sut.ac.th). namely actual cost data collection and variance analysis. The actual cost data that are collected are used as input of the analysis. In practice, there are a number of the actual cost data. Most of them are in paper-based format and irregularly received during the construction phase. The collection requires tedious effort and these data need to be re-keyed. The collection also needs to be on-time and up-to-date otherwise these data will lose their usefulness. This process usually fails the whole cost control task. The data re-keying increases errors that can consequently cause errors in the analysis. Therefore, important barriers to the cost control are the transformation of the cost data format from paper-based into machine-readable data or the data re-keying task. This task is very painstaking and time consuming. It usually makes these data obsolete or they arrive after the completion of the construction work. The project costs cannot be controlled effectively. The major obstacle to monitoring and controlling the progress of the project is the time and effort for data collection [10]. That makes the tracking and controlling tasks are not cost effective. In addition, if the data collection is carried out manually, it will result with a lot of errors and delays.

Small and medium-sized contractors (SMCs) suffer from this problem since they have limitations on investment and personnel [3]. SMCs normally do not invest on the sophisticated project control software and do not have a cost control department in their companies. They need an effective cost control system particularly. SMCs are an important group of construction industry of many countries. In Thailand, they represent $99.7 \%$ of the total numbers of contractors. They generate an about $85 \%$ of the GDP (Gross Domestic Product) and $81 \%$ of the employment of the whole construction industry [11]. The development of the Barcode-based Cost Control System (BCCS) using Automatic Identification and Data Capture (AIDC) technology is described. The technology has a potential to assist the actual cost data collection and reduce errors in the rekeying process.

\section{A Cost Control of SMCs}

Survey studies were conducted on the existing cost control of SMCs. A total of 10 SMCs was selected. Their types of construction work were buildings, residential houses, and housing estate. Their project sizes were varied between 30-300 million baht. Their registered capitals were between 2-50 million baht and their working experiences were from 3 to 40 years. The study organized interview sessions with company owners or engineers who were directly responsible for the cost control task. These study cases were used to reflect common practice of cost control of SMCs. 
The findings are described here. These SMCs subcontract all or almost all labors so that their companies are in compact size and flexible with their workload fluctuation. They can transfer risks in labor costs to the subcontractors. They do not need to directly control the project labor cost. The cost control system for SMCs should focus on material costs and other expenses incurred in the construction project.

There is neither cost control department nor specialized persons to control the project costs but usually maintained by the company owners themselves. These SMCs do not have a systematic cost control system. The company owners are responsible for many other managerial tasks and do not have enough time to collect and analyze project cost data. They normally use the project BOQ (bills of quantity) of to arrange budgets and use the structure of work items listed in BOQ as the cost centers. There is no cost accounting system to collect actual cost data incurred. They collect bills or invoices instead. Some companies (of the study cases) cannot collect all these documents and lose some of them in a mess. Only two companies use expense journals to manually record all actual cost data from the invoice documents. However, the posting dates do not have a regular interval and long delays from the transaction dates (the dates when expenses are recognized). The expense journal does not classify these cost items into proper cost centers so that a variance for each particular cost centers cannot be analyzed and identified. It can only be used to determine the variance of the total cost. Their existing cost controls are not effective.

Although these SMCs realize the importance of cost control system, the actual cost data collection and the re-keying tasks are very tedious, time-consuming, and prone to errors. It is difficult to maintain these tasks continuously and on time. A practical cost control system for SMCs should be developed with a tool that can assist these critical tasks. The system should be capable of handling a large amount of data with great accuracy. It also should storage these data in an electronic format which can be further processed on the variance analysis and give results as quickly as real-time.

\section{Automated Data Collection in Construction}

Numerous and various information is required to successfully manage a construction project. Some of it is required immediately or quickly in real time. This includes information relating to the tracking and controlling the project [14]. Since real-time data are critical in speed, accuracy, and reliability; the collection, inputting and analyzing processes must be in very high efficiency. If this real-time system relies on people, it will be limited. The AIDC technology has been deployed in many other businesses but not yet widespread enough in construction [6]. It has the potential of higher capacity and lower prices.

AIDC is applied to the construction industry particularly on project management. RFID has an ability to identify the moving objects and it is a wireless system so that it is suitable to use in construction work site. RFID was used to trace large fabricated pipe spools on their transportation [13]. RFID had a potential to manage the information of every individual building component which has been moved to many locations during the construction process [8]. GPS has been brought to trace on the shipment of construction materials and equipment and help reduce losses and increase efficiency [9]. The video recording was used to recognize work patterns of construction workers, analyze them, and result on the project progress [1].

Barcode has a special advantage over the other AIDC because it is inexpensive and easy to use. It is the most widely used AIDC. It has been implemented on construction by some research. Barcode has been applied to check the use of construction materials by workers and accurately calculate incentives for workers from saving materials [4]. Barcode combined with Geographic Information System (GIS) has been used to track the progress of the precast building construction project [5]. Barcode was also used with Personal Digital Assistant (PDA) to the manage construction materials, labor, and tracking the progress of the construction [12], [15].

Previous research implemented many types of AIDC technology on the construction. Most of this focused on the new innovation of the technology but it never considered the readiness of the users who might be SMCs. Since SMCs are actually the majority group of the industry and still far lag behind these high technologies especially in the developing countries. However, previous research also showed that the barcode technology can be effectively used to collect data of construction. The one-dimensional barcode technology is chosen as a tool to collect and input all actual cost data of a construction project. Being cost-effective technology, barcode is suitable with SMCs. The developed system is expected to be practical for SMCs which have restrictions on investments and personnel. The development of the cost control system and the system evaluation with the real study cases are described in the next section.

\section{BCCS MODEL DEVELOPMENT}

\section{A. Development Tools}

Barcode is a symbol system that represents characters. It can be read by an optical reader and results in electronic data. One-dimensional barcode use symbols including parallel solid lines and line spacing with different widths. Symbology of one-dimensional barcode has several standards. In this study, the Code 39 standard is chosen because it is very widely used and can be read by any common reader. It is a discrete, bi-directional, self-checking, and variable-length code. Code 39 can also encode alpha-numeric characters. A Code 39 tag can be easily prepared using a special font type. Spreadsheet software, Microsoft Excel, is used to develop the BCCS model. From the survey study, the SMCs have already owned and been familiar with it while the database management software is not commonly used as much. Previous research [7] has also used the spreadsheet software to develop simple and practical information systems for construction companies. Microsoft Excel supports embedded programming. It features Data Validation which can be used to check the format and type of data input, and to maintain data consistency and integrity. It also provides the user access management for data protection and security.

\section{B. BCCS’ General Cost Centers}

The project BOQ is a main source of budgeted cost data. Construction BOQs which provide details of project budgets 
are generally categorized into different levels, namely "work disciplines", "constructing components", and "work items". For examples: structural work > first floor > formwork. Although project BOQs are unique, they consist of common construction "work items" such as formwork, rebar work, concrete work, etc. The combination of these common "work items" makes them unique. A "work item” which is the finest level of the BOQ is quantified and quoted with a price. However, a single "work item" can be composed of many "material items". For example: a "work item”, formwork, is composed of many sizes of timber, plywood, and nails. Sometimes, one "material item" can be a constituent of many "work items", for example, a rebar with dia.16 mm. can be used in floors, beams, or columns. This complex structure of a BOQ makes it difficult to track the actual costs.

Bills are the main source of the actual cost data but they give details and specify costs in "material items". The actual cost data are obviously incompatible with the budgeted cost data. They are received in a different level of details. They cannot be directly compared. However, it is more suitable to use "material items" as cost centers (units of comparison) rather than "work items". Since the actual cost data can be extracted from bills straight away, there is no need to classify this "material item" belong to which "work item".

BCCS is designed to use the General Cost Centers which is categorized into different levels, namely "constructing components", "material types", and "material items". The General Cost Centers is a material-oriented breakdown structure while the project BOQ is a work-oriented one. Fig. 1 shows the relationships and structures of the project BOQ, the General Cost Centers, and bills. The General Cost Centers can be accumulated from the company's historical projects and it is continuously generalized to cover as many work items as possible. Common "material items" can be shared from previous projects to another new project. The General Cost Centers works as a template for the new project to prepare its own budgeted costs. A new cost center can be appended on but for the cost centers that do not apply on the new project will be left their quantities to zero instead of deleting them.

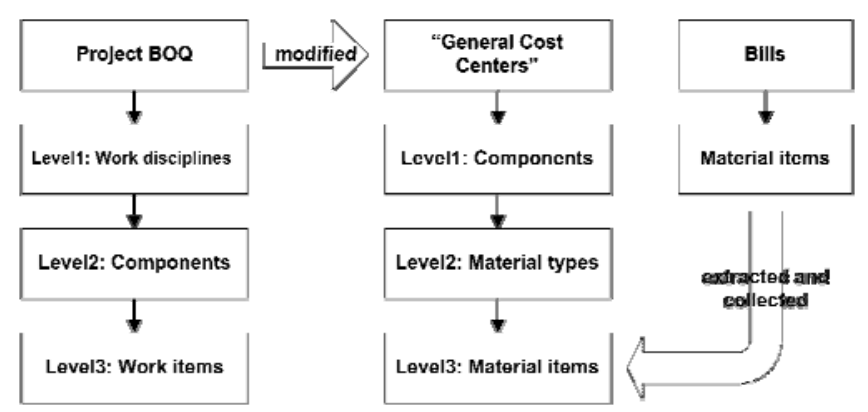

Fig. 1. A structure of the general cost centers

Each cost center is assigned with an individual cost code. The coding system is divided into three levels, namely work discipline, material type, and material item. Each level is assigned with two digits, giving a total six digits, and one alphabet "A". All cost codes of the General Cost Centers are converted into barcodes using Code 39 standard. They are sorted by titles of the cost centers in an alphabetical order. The printout of this list will be used with the barcode reader when inputting actual cost data of these cost centers.

\section{Functionality}

BCCS includes four modules, namely 'Budget Database”, “Actual Cost Form”, “Actual Cost Database” and "Report”. They are used for preparing budgeted costs, inputting actual costs, recording actual costs, and reporting results, respectively. Each module works on a separated Worksheet. These modules can retrieve and share some information each other. Fig. 2 shows the connections and the working processes of these four modules of BCCS.

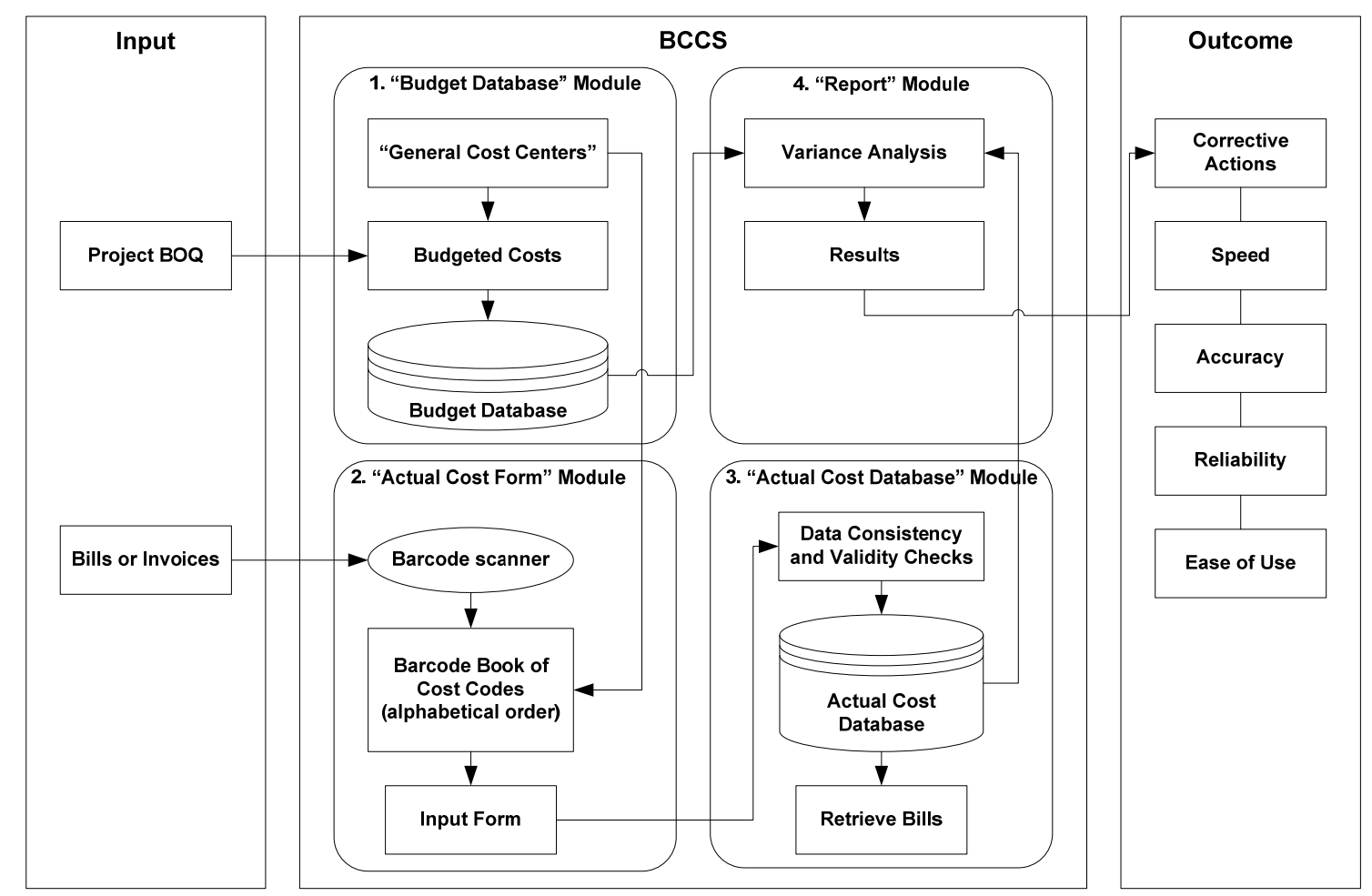

Fig. 2. A schematic diagram of functionality of BCCS' modules. 
The "Budget Database” module works when a new project arrives. It is used to arrange all budgets of the new project. These budgets are arranged in the existing General Cost Centers. The Budget Database consists of three groups of data fields. The first group of data fields such as Cost Code (primary key), Cost Center, Unit Name, Unit Cost, Quantity, and Item Sum. This group of data fields is originally taken from the project BOQ. The second group of data fields is automatically generated, such as Current Unit Cost, Actual Quantity, Actual Cost To-date, and Status. These data fields refer to the collected actual cost data. The Status data field indicates the current status of each cost center whether it is overrun or underrun.

The "Actual Cost Form" module is designed to facilitate input from bills. This module works with a barcode optical scanner to transform paper-based actual cost data into electronic data. All bills or invoices received from venders, or subcontractors are used as main source documents of all actual costs. The Worksheet is arranged to imitate the common layout of these bills. It is used as an input form. Various expenditure items (are usually expressed in terms of construction materials) can be recognized as appeared on the General Cost Centers. Apart from that, a book of cost codes is prepared and printed out. These cost codes are sorted in an alphabetical order of cost centers for a quick lookup. The cost codes alongside with their own barcode images are used with the scanner to automatically identify and retrieve information of the particular cost center needed. With helps from barcodes users can rapidly and neatly input actual cost data from the bills. Unit costs of cost centers can be updated according to the bills. Any data inputted can also be first validated and alert users to correct if any inconsistency is found.

The "Actual Cost Database" module works right after the "Actual Cost Form” module. After information on a bill is filled in the form, it is passed to the Actual Cost Database module which records this information on the database-like Worksheet. Information of a bill is stored as one data record of the database. There are many data fields which have already been filled in the form such as Form ID Number, Date, Cost Code, Quantity, Current Unit Cost, Note, and Item Sum. The "Form ID Number" is the primary key. Finally, important data of all paper bills can be safely recorded in an electronic format on the database.

The "Report" module processes data on the Budget Database and the Actual Cost Database and presents useful results for decision making. Results are reported as the variances between the budgeted cost and the cumulative actual cost to-date. The Report shows the comparison results by groups of the work discipline level (the top level of the breakdown structure). Also, results of the current variances along the project timeline are reported. Users can receive the current actual performance against the plan in different levels of details. Users should be able to discover any problem right on-time and to make proper corrective actions effectively.

\section{RESUlts}

BCCS is tested with two construction projects of a SMC. These projects have already been completed in 2008. They had total budgets of 40.7 and 41.5 million baht (Project A and
$\mathrm{B}$, respectively). They are selected as test cases because cost data of these projects have been well recorded and this SMC is willing to participate. The cost data including the project BOQs and bills are carefully kept and they are manually re-written on expense journals by the company owner herself. Each project has about 300 bills and about 700 billing items. The aspects of speed, accuracy, and reliability of BCCS are put to the test. The experiment is designed to re-input all cost data of the projects using BCCS and to compare the results from BCCS with the manual method one project at a time. The project cost data are re-inputted twice for each project. This is to check the reliability of the inputting process of BCCS. Firstly, the project BOQs are modified into the General Cost Centers as the project budgeted costs and their data are stored in the "Budget Database". The project bills are inputted through the "Actual Cost Form” using barcodes of the cost centers. The actual costs of these projects then are transformed and saved into the "Actual Cost Database". Time required for the data inputting process is obviously faster and it is more pleasurable than of the manual method. There is no discrepancy between the two inputting sessions for both projects. This means the inputting process of BCCS is reliable.

For Project A, as recorded on the expense journal, it had the overall actual cost $24.3 \%$ less than the budget (underrun) while the result from BCCS shows the overall actual cost is $17.9 \%$ less than the budget (underrun). There is a $6.4 \%$ difference between the two methods. For Project B, there is also a $4.1 \%$ difference between the two methods. The further investigation discovers the items causing mistakes in the expense journals. These mistakes come from manual calculations and numerical typos. This difference can mislead the true performance (profit/loss) of the project. This test case indicates that the company actually gains gross profit from Project A less than it has realized. The company owner admits that some errors might occur on her recordings because there are a number of data and figures. BCCS can efficiently handle these data and reduce human mistakes. She is also convinced that BCCS can help reduce time and effort for the cost control task.

\section{CONCLUSION}

SMCs are a major group of the construction industry in many countries. However, they are low-technology and full of development restrictions. Although these SMCs realize the importance of cost control system as the project costs can directly affect the profits of the business, their cost controls are mostly manual and inefficient. This is regarded as a very high priority problem needed to be solved. A large amount of paper bills is piled up and overwhelms the project managers. Their cost controls cannot synchronize with the project progress. The cause of failure of their cost control task is pinpointed especially in this study and leads to the development of Barcode-based Cost Control System (BCCS).

The barcode which is commonly used in other industries but construction can also benefit SMCs. BCCS facilitate the actual cost data collection. It helps control the project costs in a level of details which never been seen before. It can keep tracking the overrun status of the cost centers up to the second 
level or the "material types”. It is useful for on-time decisions making and corrective actions. BCCS can improve the accuracy of the data comparing with the existing manual method and give reliable results. The concept of the General Cost Centers is introduced in the study. The General Cost Centers are implemented to share common "material items" across projects. They can ease up the project budget preparation and the actual cost collection since the users do not need to classify any expenditure items received.

The future research and development can extend BCCS to the preceding and the succeeding tasks, for example collaboration with suppliers, materials inventory management, and worker-timekeeping.

\section{REFERENCES}

[1] J. Abeid, E. Allouche, D. Arditi, and M. Hayman, "PHOTO-NET II: a computer-based monitoring system applied to project management," Automation in Construction, vol. 12, pp. 603-616, Oct. 2003.

[2] S.H. Al-Jibouri, "Monitoring systems and their effectiveness for project cost control in construction," International of Project Management, vol. 21, pp. 145-154, Mar. 2003.

[3] V. Benjaoran, "A cost control system development: A collaborative approach for small and medium-sized contractors,” International Journal of Project Management, vol. 27, pp. 270-277, May 2009.

[4] Z. Chen, H. Li, and C.T.C. Wong, "An application of bar-code system for reducing construction wastes,” Automation in Construction, vol. 11, pp. 521-533, Oct. 2002.

[5] M.Y. Cheng, and J.C. Chen, "Integrating barcode and GIS for monitoring construction progress," Automation in Construction, vol. 11, pp. 23-33, Jan. 2002.
[6] I.N. Davidson, and M.J. Skibniewski, "Simulation of Automated Data Collection in Buildings," Journal of Computing in Civil Engineering, vol. 9, pp. 9-20, Jan. 1995.

[7] T. Hegazy, and T. Ersahin, "Simplified spreadsheet solutions I: Subcontractor information system," Journal of Construction Engineering and Management, vol. 127, pp. 461-468, Nov. 2001.

[8] Y. Junichi, A. Eiji, and A. Tatsuo, "Parts and packets unification radio frequency identification (RFID) application for contruction," Automation in Construction, vol. 14, pp. 477-490, May 2005.

[9] H. Li, Z. Chen, L.Yong, and S.C.W. Kong, “Application of integrated GPS and GIS technology for reducing construction waste and improving construction efficiency," Automation in Construction, vol. 14, pp. 323-331, May 2005.

[10] R. Navon, "Research in automated measurement of project performance indicators," Automation in Construction, vol. 16, pp. 176-88, Mar. 2007.

[11] Office of Small and Medium Enterprises Promotion (OSMEP). (September 2008). The White Paper on SMEs of Thailand in 2007 and Trends 2008, Available: http://cms.sme.go.th/cms/web/osmep/home.

[12] S.W. Oh, H.J. Chang, Y.S. Kim, J.B. Lee, and H.S. Kim, “An application of PDA and Barcode technology for the improvement of information management in construction Projects," in Proc. of 21st International Symposium on Automation and Robotics in Construction, Jeju, Korea, 2004, pp. 518-524.

[13] J. Song, C.T. Hass, C. Caldas, E. Ergen, and B. Akinci, “Automating the task of tracking the delivery and receipt of fabricated pipe spools in industrial project,” Automation in Construction, vol. 15, pp. 166-177, Mar. 2006

[14] G. Stukhart, Construction Materials Management, Marcel Dekker, New York, 1995.

[15] H.P. Tserng, and R.J. Dzeng, "Mobile construction supply chain management using PDA and Bar Codes,” Computer-Aided Civil and Infrastructure Engineering, vol. 20, pp. 242-264, Jul. 2005. 